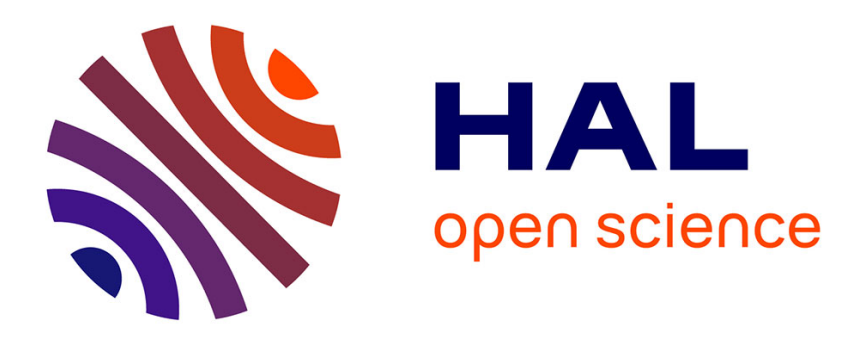

\title{
Calcul du bruit de jet par une approche semi-déterministe
}

F. Bastin, Ph. Lafon, S. Candel

\section{To cite this version:}

F. Bastin, Ph. Lafon, S. Candel. Calcul du bruit de jet par une approche semi-déterministe. Journal de Physique IV Proceedings, 1994, 04 (C5), pp.C5-955-C5-958. 10.1051/jp4:19945209 . jpa-00252893

\section{HAL Id: jpa-00252893 https://hal.science/jpa-00252893}

Submitted on 1 Jan 1994

HAL is a multi-disciplinary open access archive for the deposit and dissemination of scientific research documents, whether they are published or not. The documents may come from teaching and research institutions in France or abroad, or from public or private research centers.
L'archive ouverte pluridisciplinaire HAL, est destinée au dépôt et à la diffusion de documents scientifiques de niveau recherche, publiés ou non, émanant des établissements d'enseignement et de recherche français ou étrangers, des laboratoires publics ou privés. 


\title{
Calcul du bruit de jet par une approche semi-déterministe
}

\author{
F. BASTIN, Ph. LAFON* et S. CANDEL ${ }^{* *}$
}

SNECMA, Département YKV, Centre de Villaroche, 77550 Moissy-Cramayel, France

* EDF/DER, 1 avenue du Général de Gaulle, 92141 Clamart cedex, France

** Ecole Centrale de Paris, Laboratoire EM2C, 92295 Châtenay-Malabry cedex, France

\begin{abstract}
The whole proceeding of the evaluation of turbulent jet noise with Lighthill formulation from large scale instationnary aerodynamic data is presented. The basic case of the two-dimensional shear layer is completely studied and the main features of the method are established. The far field acoustic fluctuations show a satisfying spectral contents and the directivity can be compared to what is observed for subsonic jets. The first aerodynamic data of the plane jet are also presented. They should allow more precise acoustic validations before the more realistic case of the axisymmetrical jet.
\end{abstract}

\section{Principe de la méthode}

L'analogie de Lighthill permet d'exprimer le champ acoustique rayonné par un écoulement comme la solution d'une équation de propagation en espace libre où l'influence des fluctuations turbulentes est ramenée au terme source, à savoir la double divergence du tenseur de Lighthill $T_{i j}$ [4]. Pour des fluctuations isentropiques et à haut nombre de Reynolds, $T_{i j}$ se ramène à $\rho u_{i} u_{j}$ et le formalisme des fonctions de Green conduit à la formulation classique

$$
\rho^{\prime}(\mathbf{x}, t)=\int_{V} \frac{\partial^{2}}{\partial y_{i} \partial y_{j}}\left[\rho u_{i} u_{j}\left(y, t-\frac{|\mathbf{x}-\mathbf{y}|}{c}\right)\right] \frac{d \mathbf{y}}{4 \pi c^{2}|\mathbf{x}-\mathbf{y}|}
$$

où $\rho^{\prime}$ représente la fluctuation de densité due à la présence du volume $V$ de sources turbulentes.

Pour les cas d'écoulements libres et fortement turbulents qui nous intéressent, le calcul complet, par simulation directe, du champ aérodynamique instationnaire reste inabordable avec les moyens numériques actuels et l'approche classique du problème consiste à estimer l'intégrale (1) en évaluant les tenseurs de corrélation nécessaires à partir d'une connaissance statistique du champ turbulent. Nous optons pour une démarche intermédiaire où seules les fluctuations cohérentes du champ turbulent sont calculées. Effectivement, plusieurs études $[3,5]$ tendent à montrer que le rayonnement acoustique d'un jet est fortement déterminé par les grosses structures de l'écoulement tandis que les fluctuations aléatoires de la turbulence à fine échelle ne se traduisent que par un faible fond sonore large bande. Ainsi, nous adoptons une approche de type semi-déterministe où une moyenne statistique du champ aérodynamique est calculée. L'écoulement obtenu reste instationnaire mais seules les fluctuations cohérentes sont conservées. 


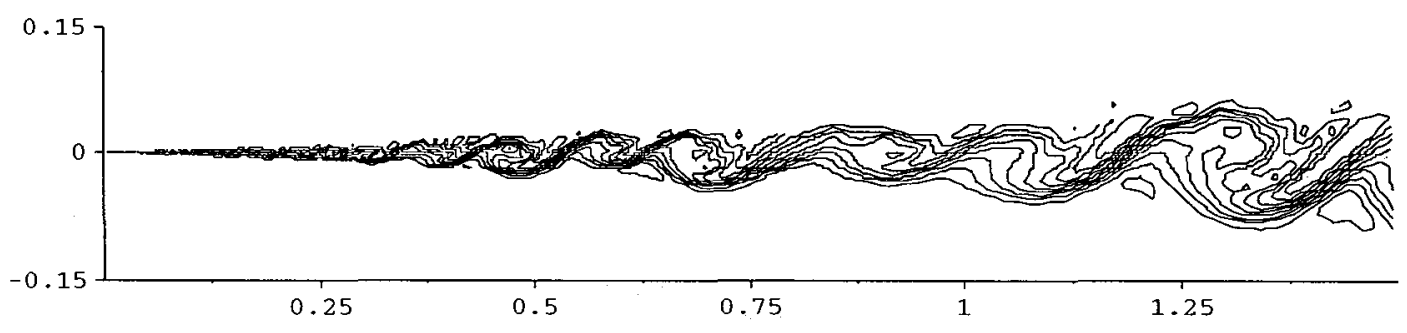

Figure 1: Diffusion d'un scalaire passif dans une couche de mélange $M_{1}=0,7 \quad M_{2}=0,3 \quad M_{c}=0,19$.
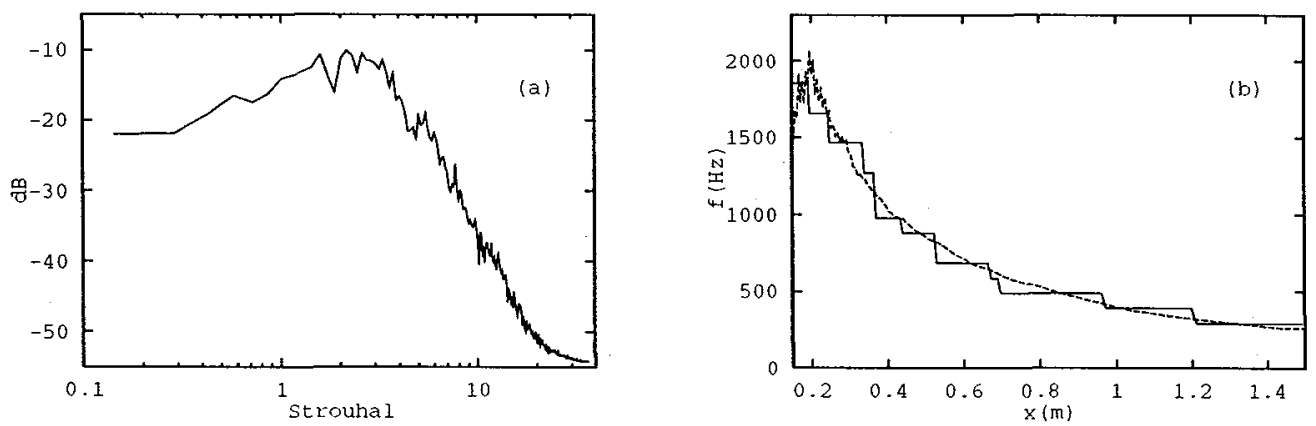

Figure 2: Fluctuations aérodynamiques (a) Spectre de $u$ pris en $\mathrm{x}=0.3 \mathrm{~m} S t=f U_{c} / x$. (b) Pic spectral — calcul - - - théorie des instabilités linéaires.

\section{La couche de mélange}

Dans la pratique nous utilisons un modèle $k-\epsilon$ compressible standard, mis en œuvre dans le code bidimensionnel KAOLIN. Les variables calculées sont $\rho, \rho u, \rho v, E, \rho k$ et $\rho \epsilon$ où $\rho$ est la densité, $u$ et $v$ les deux composantes de la vitesse, $E$ l'énergie totale par unité de volume et $k$ et $\epsilon$ sont l'énergie cinétique et la dissipation turbulente. Le schéma numérique utilisé est celui de Mac-Cormack à l'ordre deux qui est un prédicteur correcteur explicite d'ordre deux en espace et en temps. La précision du schéma semble importante pour obtenir des résultats instationnaires. Les conditions aux limites sont de type caractéristique.

Le premier écoulement traité est la couche de mélange à deux dimensions, pour lequel les validations aérodynamiques possibles, aussi bien par des modèles théoriques que par des résultats expérimentaux, sont nombreuses. De plus le mécanisme fondamental du développement d'un jet est l'interaction des couches de mélange issues de la buse.

Pour une couche de cisaillement subsonique-subsonique avec $M_{1}=0,7$ et $M_{2}=0,3$ on obtient l'écoulement présenté figure 1 où on voit nettement les structures cohérentes classiques de ce type d'écoulement [1]. Le spectre des fluctuations de vitesse longitudinale est satisfaisant (figure 2) et on vérifie le très bon accord du contenu spectral de ces fluctuations avec la théorie classique des instabilités linéaires [2].

A partir de ces résultats aérodynamiques, nous calculons l'intégrale de Lighthill pour obtenir les fluctuations acoustiques en champ lointain. Le spectre des fluctuations acoustiques (figure 3 ) est 

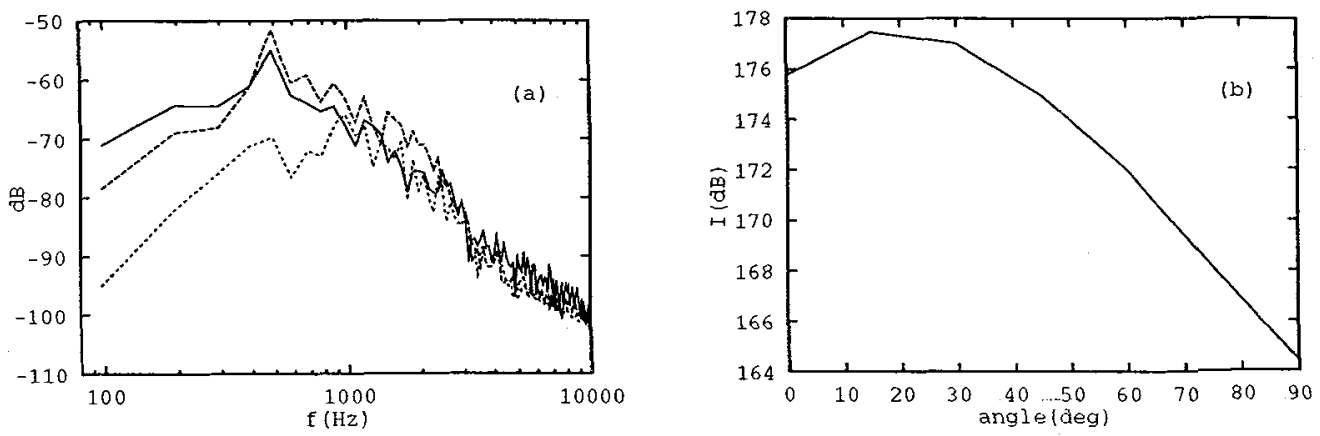

Figure 3: Résultats acoustiques (a) Spectre $-\theta=0^{\circ}---\theta=45^{\circ}$ $\theta=90^{\circ}$

(b) Directivité normes SAE adaptées.

satisfaisant en ce sens qu'on retrouve bien les différentes fréquences présentes dans l'écoulement. Il paraît difficile de valider le niveau obtenu mais le diagramme de directivité peut-être comparé à celui d'un jet de même nombre de Mach convectif.

\section{Le jet plan}

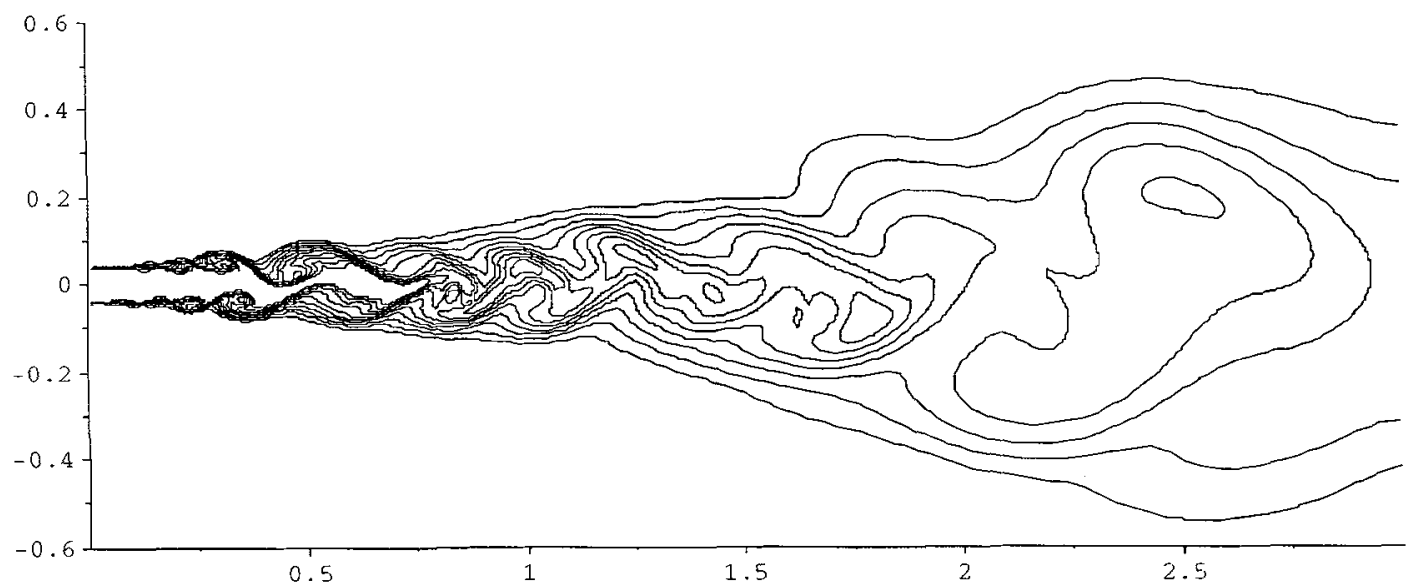

Figure 4: Diffusion d'un scalaire passif dans un jet plan $M=0,5 \quad D=7,5 \cdot 10^{-2}$. Départ de la zone de sortie en $x=1,5 \mathrm{~m}$.

Le jet bidimensionnel est un cas plus réaliste, surtout dans la perspective d'arriver au jet axisymétrique. Le calcul se fait grossièrement de la même façon que pour la couche de cisaillement pure. Pour simplifier les problèmes de conditions aux limites, on a rajouté une zone de sortie où la résolution $k-\epsilon$ est suivie d'une étape de diffusion. La condition de stabilité impose un pas de temps assez fin de l'ordre de $10^{-2} \mathrm{D} / U$. Le développement du jet pour $M=0,5$ est très réaliste (figure 4). Là encore le spectre des fluctuations aérodynamiques est fiable et devrait permettre d'effectuer 
des calculs acoustiques dans de bonnes conditions. L'un des problèmes qui se pose est la nécessité de capter aussi bien les hautes fréquences des instabilités du début des couches de mélange que les fréquences plus basses dans la zone développée qui sont probablement les plus importantes quant au rayonnement acoustique.

\section{Conclusion}

Le cas de la couche de mélange a permis de mettre au point dans les détails une méthode originale d'estimation du bruit de jet. Le problème délicat de l'obtention des instabilités convectives de l'écoulement tout au long de son développement avec un modèle statistique est maintenant bien cerné. L'utilisation correcte de la formulation de Lighthill dans ce cadre semi-déterministe a été étudiée. Les mêmes calculs sur le cas du jet plan puis axisymétrique devraient permettre une validation plus précise de l'ensemble de la méthode.

\section{References}

[1] Brown G. L. and Roshko A., J. Fluid Mech. 64(4) (1974) 775-816.

[2] Ho C.-M. and Huerre P., Ann. Rev. Fluid Mech. 16 (1984) 365-424.

[3] Laufer J. and Yen T.-C., J. Fluid Mech. 134 (1983) 1-31.

[4] Lighthill M. J., Proc. Roy. Soc. A 211 (1952) 564-587.

[5] Seiner J. M., McLaughlin D. K. and Liu C. H., Nasa Technical Paper 2072 (1982) 